\title{
Applied CAL on Problem Based Learning Using Gagne's Instructional Design
}

\author{
http://dx.doi.org/10.3991/ijet.v9i9.4116 \\ Sri Sundari ${ }^{1}$, Harsono ${ }^{2}$, Titi Savitri P. ${ }^{2}$, and Ova Emilia ${ }^{2}$ \\ ${ }^{1}$ Universitas Muhammadiyah Yogyakarta, DI Yogyakarta, Indonesia \\ ${ }^{2}$ Universitas Gadjah Mada, DI Yogyakarta, Indonesia
}

\begin{abstract}
In the Problem-Based Learning (PBL) model, students are expected to study independently. One of the methods that can improve the ability or skill of learners is using Computer Assisted Learning (CAL). Implementation of CAL in PBL should be able to create Self-Directed Learning (SDL) culture through appropriate instructional design and interesting modules. In this paper, the CAL software is developed using multimedia learning principles, convenient appearance, and user-friendly navigation. The CAL's learning content is designed using Gagne's instructional design. The experiment proved the CAL was able to give effect size 0.89 and developed self-directed learning culture. From the interviews, students were very glad and interested to use the CAL modules because they can learn anytime and can reach the course objectives without a lecturer.
\end{abstract}

Index Terms-Problem Based Learning, Computer Assisted Learning, Self-Directed Learning, Gagne's Instructional Design

\section{INTRODUCTION}

In an e-learning implementation, it takes a supporting technology that acts as the primary learning devices. Today, the computer is a major supporting device of elearning. There are two roles of computers in e-learning: in the Computer Based Learning (CBL), computer is the central part of the learning, whereas in the Computer Assisted Learning (CAL) computer merely as one of learning tools or computer-conveyed.

The main characteristic on CAL is self-paced learning (in accordance with the conditions and speed of learning of each student). CAL is student-centered learning, because the students who have the autonomy should decide what to learn, how to learn, learning through what, and where they will learn; while the task of educators is as a facilitator for all combinations, optimal learning modalities can be run effectively. So, CAL has the potential to build a culture of SDL.

In PBL, students are expected to learn independently (self-directed learning) so it is necessary to develop a method of learning that can improve the ability of students to perform learning without the assistance or intervention of lecturers. CAL is one method of learning that is considered suitable for PBL, but must equip with a proper instructional design for building self-directed learning, one of which uses Gagne's instructional design. Thus, the question in this paper is how to apply the Gagne's instructional design on CAL for enhancing the student analytically skills in PBL?

\section{DEVELOPMENT CONCEPT}

\section{A. Computer Assisted Learning}

CAL has distinctive characteristics: focus on independence in learning, providing an interactive learning tool, and providing guidance [1]. CAL has parts that construct a unique structure; these parts are the software requirement, software design, the software using, and content, message of the knowledge, and supporting software. The CAL module should contain text, audio, video, graphics, etc. In the lecture point of view, the CAL module should easy to use (either the manufacture or utilization), requires minimal training, allowing the desired learning, and enabling learning control according to the environment. While, from the student point of view, CAL module should flexible, has richer learning materials than face-to-face, running on a computer that is already available, allowing adequate collaboration, and including the development of advanced materials through class or group discussions.

Using CAL on learning, particularly in PBL has benefits [2], as follows:

1. Delivery of lessons becomes more standardized, so that every student who reads or hear through the presentation through CAL receive the same message,

2. Teaching becomes more attractive because they function as the central focal point and cause the students give attention,

3. Provides an interactive learning

4. Shorten the learning time

5. Teaching is giving when necessary or desirable,

6. The positive attitude of the students towards what they learned could enhance, included the culture of self-directed.

From the above explanation that CAL has benefits as a tool that can attract attention, provide experiences for students to interact directly to the learning material so as to create a positive attitude of the students to learn independently.

\section{B. Multimedia cognitive learning theory}

In cognitive psychological theory, there is a working memory. This memory consists visually, and auditory working memory that works separately [3] and both has a very limited capacity [4][5].

Cognitive load theory explains that humans have the two separate systems in verbal and visual information presented [6]. The dual coding theory explains that the learning process would be meaningless if the student selects and store irrelevant information, and then organize 
the information stored into something coherent and connect them to be more meaningful information [7].

The internal, external, germane cognitive load is physiologic terminologies that associated with the memory management [8][9]. The level complexity of the course material causes the intrinsic cognitive load, while the technique how to presenting the learning material cause external cognitive load [10]. The intrinsic cognitive load cannot manipulate because it is the character of the interactivity of the elements in the material; the intrinsic cognitive load is fixed. However, the extrinsic cognitive load can be manipulated.

If the presentation of the course material correct, it will cause students easily to understand and reduce their extrinsic cognitive load. A learning material with a high level of intrinsic load if given in a good manner, then the cognitive processes in working memory will run smoothly. In contrast, although the intrinsic cognitive load is low, but if presented in a wrong manner, then the cognitive processes in working memory will run slow or even stop altogether.

CAL design should consider the limitation of working memory capacity and should consider the verbal and visual working memory that work separately. Dual processing theory states that the presentation of information through multimedia instructional will be meaningful if the information received is selected first before delivered to the student. The information should be arranged and interconnected to each other. To cope with this condition in the delivery of learning materials on CAL should use the above principles; the information should be arranged and coherently.

Besides the cognitive load theory and dual processing theory, in cognitive multimedia learning theory, there is a constructivism learning theory. Due to the CAL instructional design, the constructivism learning theory suggests that:

1. Students should be encouraged actively to construct their knowledge; it is within their self-directed learning principles.

2. So that the students can understand and apply the knowledge, they should learn how to solve problems and find everything for their experience.

3. Learning is the process of building knowledge, not just absorb or remember knowledge.

4. Learning is a process of constructing knowledge that is always changing continuously through assimilation and accommodation of new information.

Implications of the theory of constructivism to the design of CAL to establish a culture of self-directed learning is:

1. Focusing on the thinking ways or mental processes of students.

2. Giving priority to the role of students in their initiative as well as encourage students to actively engage in learning activities

3. Designing learning begins from the simplest to the most-complicated thing in stages

When defining a multimedia learning, it should distinguish three components contained in it, namely: media, model, and modality. Media translated as systems used to deliver messages or instructions such as books or comput- ers. The model defined as the format used in presenting information or tasks include writing or drawing while the modality assumed as the channel used by the students to process the information received (auditory or visual).

\section{Gagne's Instructional Design}

CAL in order to be effective, in accordance with the concept of multimedia learning, instructional design should be developed so that can foster a culture of selfdirected learning. One of the instructional designs of learning to be effective is the nine instructions [11], namely:

1. Gain attention,

2. Describe the goal,

3. Stimulate prior knowledge

4. Present the material will be learned,

5. Provide learning guidance

6. Obtain performance,

7. Give feedback,

8. Assess the learning performance, and

9. Enhance retention and transforming.

The application of Gagne's nine instructional designs on CAL described as follows:

1. Gain attention: attract the attention of students by using images, sound, information, text, and use contrasting colors as a background screen. Animation can be added as well as a particular part to stimulate the attention of students. At the beginning of the CAL can be shown the main question regarding the learning material and how students can answer it.

2. Describing the goal: the CAL's learning objectives displayed sequential and clearly. The title of learning also written as it will provide early clues about the content of learning. In addition, the learning objectives are presented using an interesting way to raise the motivation of students participates in learning activities.

3. Stimulate prior knowledge: it is one technique to generate short-term memory; this can be done by giving a test or question regarding the knowledge that discovered, related to the learning that will be carried out. It can also be done by showing a picture or animation that can inform the relationship between the knowledge that acquired and the skill that pursued.

4. Present the learning material: the main point of this is how to get a response from the student. The presentation of the learning material must be able to generate the stimulus. Therefore, the content view should be clear, simple and straight to the point of discussion to explain concepts. Images, sound, video and animation elements can be used to illustrate ideas, show, and present the content. The interface also provides navigation tools for accessing a particular theme. The teaching materials should easily accept by the students; it is necessary to recognize the information processing that occurs when learning delivered through multimedia.

5. Provide learning guidance: learning coverage and objectives are shown using a concept map and short video.

6. Obtain performance: the results of the previous learning should show on the initial interface and the 
results of the current study show at the end of the CAL interface.

7. Give feedback: students were given feedback after learning a topic to be more motivated. Feedback is positive comments after successfully answering a quiz or a clue if the answer.

8. Assess the learning performance: on each topic consist a tutorial and at least a quiz. The quizzes use for measuring the learning performance.

9. Enhance retention and transformation: CAL shows a summary to stimulate the ability to remember the concept, and then gives a test to measure how much a student can apply the gained knowledge. This method used so that students can transfer or use of the knowledge, skills, and strategies when faced with new problems and situations.

\section{Methodology}

This study intended to solve practical problems in teaching-learning that need to be remedied crucially through class action [12]. The class action steps consist of learning investigation, an action plan, assessment, and treatment. To prove the purpose of the research, it needs to determine the research elements, including the subject, the research variables, and how to analyze the data.

\section{A. Subject}

Based on the investigation and evaluation of problembased learning in medical school, it is known that the lowest graduation number is in alimentary block compared to other blocks. Thus, the research samples were 249 students of alimentary block. The students who are willing use CAL module as the experimental group and other students are entering as a control group.

\section{B. Variables}

The research variables consisted of analytical skill of students as dependent variable and the effect of step-bystep instructional learning as independent variables. The analytical skill obtained at the end of the course indicated by a score of CAL. The independent variable of CAL based on the nine steps Gagne's instructional.

\section{Method}

The research divided into four steps "Fig. 1": requirements, structures, core, and the result. The research has a goal to implement CAL in PBL using Gagne's Instructional Design to enhance the analytical skill of students as indicated by an increase in test grade. The structure of the research includes the design, implementation, and evaluation.

\section{Analysis}

This research is a classroom action research for the development of a learning model, so that uses descriptive qualitative analysis. The effectiveness of the CAL model for student analysis ability performed using t-test and effect size calculation.

\section{RESUlT AND DisCUSSION}

\section{A. Result}

No subject grouping by sex, age, or origin, but the students divided into a control group and an experimental group based on activities uses CAL module. If students



Figure 1. Research method steps

using CAL included in the experimental group and vice versa. There are 249 students in the sample that divided into two general categories: 203 students as the experimental group and 46 students as a control group.

From the statistical analysis, it is known that there is no difference between the mean pretest score control group with the experimental group. These results indicate that the two groups have the initial knowledge homogeneous. The mean of posttest scores of between the two groups had statistically significant differences. The result indicates that after using CAL, the mean of alimentary posttest scores of the experimental group was higher than the control group. Statistically, the more often students use the CAL student posttest score will be higher. The effect size of the CAL was 0.89; it is indicating that the learning to use CAL has a high effect on the posttest score.

Based on the analysis, all the correlation coefficient is positive; there is a relationship between the independent variables with the frequency of use CAL. From the analysis also known that better learning content, more attractive in appearance and easier the using of navigation increase the student interest and finally more often learners to use CAL.

\section{B. Discussion}

From the Focus Group Discussion (FGD) note that the use of the internet facility or power point is less effective, because: the presented material does not draw attention, does not help learners to understand the material, and does not support to evaluate the learning performance. The elearning environment also is not present in a systematic manner. CAL in this study was developed using the principles of cognitive load and human-computer interaction (HCI), and it proved that the CAL is effective for delivering the teaching materials.

The diarrhea module of CAL (Alimentary block) proved as effective tools because the learning outcome of experiment group is increase. The effectiveness of this is possible because of the development of learning models tailored to the needs of the students, including the needs of self-directed learning; CAL is also developed by using the principles of multimedia development. It is also in 
PAPER

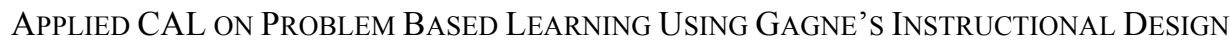

accordance with other studies that suggested that the presentation of the material with text accompanied by illustrations that more preferred than text only and dynamic visual display is more preferred than static. The use of animation will reduce cognitive load. In addition, task or exercise is used to accelerate the understanding and remembering of concepts [13].

Constructive cognitive load is the cognitive load caused by cognitive processes that are relevant to the understanding of the material and the process of construction knowledge. If working memory filled with intrinsic and extrinsic cognitive load and there is no place for constructive cognitive load means the worker memory can not organize, construct, encode, elaborate or integrate the learning material as well the knowledge stored in longterm memory. The information is presented using the wrong technique is difficult to understand. Such information may successfully save in long term memory, but it may be difficult to be called back because not connected with the relevant knowledge.

Constructive cognitive processes occur automatically if there is an empty working memory as a result of minimal intrinsic and extrinsic cognitive load. However, it can be influenced by student motivation and attitudes towards the material being studied. Without motivation and a good attitude towards the learning process, although the material is well managed, the learning outcomes will not maximized.

The implications of working memory function in designing CAL are:

1. Understand the level of complexity of the learning material or the amount of presented information;

2. Determine the level of students' prior knowledge;

3. Minimize the number of intrinsic and extrinsic cognitive load;

4. Facilitate a process to increase the constructive cognitive load (the acquisition and construction of knowledge scheme) [14].

In addition to the above theory, there are multimedia development principles that will be used to develop the CAL modules. Those principles include:

1. Multimedia principle: adding a graph to illustrate a concept can accelerate understanding. Graphs can give many illustrations, but this must be in line with the delivered message. If the illustrations using graph submitted too excessive will lower the quality of learning. The graph is also used to show the student's progress of learning.

2. Contiguity principle: words conveyed through sounds and images displayed simultaneously at one time will give a better explanation than delivered separately. Those are consistent with the cognitive theory of multimedia which states that the relationship of words and images processed together in the working memory to construct knowledge [15]. In this study, CAL is equipped with video and narration to explain the image or theory.

3. Contiguity principle: explanation of the concept better conveyed using sound instead of using the text displayed visually. Working memory has two components, namely visual and auditory so that its capacity increased if the information delivered using two modalities (audiovisual), this is better than just using one modality only [3]. CAL uses video and narration to explain the concept.

4. Coherence principle: the use of visualization, text, and sound arbitrary or excessive will harm learning. Students will learn better through related conclusions and emphasis on the word corresponding to the picture rather than through explanation is too long. A short presentation will allow students to select and organize relevant information. The duration of the video set less than ten minutes to explain the theme.

5. Redundancy principle: the learning explanation using audio and charts is provided more effective compared to the audio and text. CAL module is designed using video and narrative to explain the pictures and graphics.

6. Personalize principle: daily language used to describe a concept more easily understood by students when compared to formal languages. CAL uses video to explain the concept using a formal language, but selected the most simple and easily understood.

CAL development should consider the limitations of human existence, which is limited in focus, giving attention, and recalling.

Based on the Field Group Discussion and literature study, the alimentary block CAL that using Gagne's Instructional Design should be developed based on effective content, convenient interface, and the consistency of navigation.

\section{Content}

The goal of learning explained clearly and detail in the beginning using short video. A student should understand and master in the analytical skills of alimentary. CAL equipped with a concept map that will provide a global picture of the position of each of the materials in the alimentary cases. Concept map helps students to understand the learning material. Concept map follows the workings of the human brain how to process information, and it is more easily if in the function of the relationship. Learning materials are delivered in concepts or summary because the less meaning of information will overload the capacity of short-term memory. The less meaning of information will causes students difficult to understand the concept. The learning material divided into smaller themes to reduce the working memory load in information processing. The pull down menu designed maximum of nine rows and each row a maximum of nine words. The learning materials delivered in sequence from the concept to the application or from the concept of diarrhea, symptoms, and ways of treatment. Presentation of the material in less text, pictures, graphs, and equipped with a narrative. The presentation can package in a video or animation. The CAL's interface using a maximum of five colors with three dominant colors with contrast settings so comfortable for the students. These methods are used to facilitate recognition, attention, and sharpen the information. Important information is given particular color, thickening, or a different font size so that the students willing to give more attention to the concept, this is a means to overcome the limitations of human attention. To measure the student learning outcomes, the model used a quiz with multiple choice questions. Quizzes are giving for each topic or concept. Total quiz score uses by students to self-assessment so that they can know the progress of their learning outcomes directly. 
The number of questions that can be answered correctly can be used to provide feedback to the achievement of the overall competence of learning materials that students mastered.

\section{Interface}

The technology of CAL software that chosen should simple and well known by the students. The CAL module created using web based application so that students easily use it. The CAL interface created using a background color that contrasts to the picture for attracting the attention of students. The CAL interface designed so look as comfortable as possible for student both in terms of color, image size, layout, and contrast. Images are used according to the material presented so that the desired message conveyed easily. Animation is not used too many and complicated, it also customized with a delivered message. Important text information displayed with a thickening or a different font size so it is easily recognised by the student. Sound and image composition adjusted; the use of sound is made as natural as possible, and it is not formally, so that is easier to understand.

\section{E. Navigation}

It is selected using pictures or icons that are well known by the students, so students can use the CAL easily. The words which more important will be marked with a more striking color or font size larger or thicker. At the beginning of learning "Fig. 2", described how students can use the CAL by pressing "?" Icon. Navigation used will also help students to access desired learning materials "Fig. 3". Students can easily select or back to other learning material even when it is working on a quiz. Finally, student can see the learning progress in a result interface using bar graphs "Fig. 4". This step very important for the student to measuring their learning improvement in developing selfdirected learning culture.

From the results of the implementation of the development model of CAL is known that there is a significant difference between the posttest scores of the control group and the experimental group, even the average of posttest score of the control group is lower than the average pretest score. It showed that learners who use CAL have analytical skills better than the control group. The study proved that the CAL module has high impact to the student skill because the effect size is 0.89 ; statistically, there are $82 \%$ of students in the control group have post test scores below the mean of experimental group's posttest scores. Often or not learners in using CAL module there is a correlation with the posttest score, the more frequently the learners use CAL module then, the higher the test scores obtained.

The statistical test used to see the relationship between the content-appearance-navigation and the score. The frequency of students using CAL has a positive relationship to learning content, appearance, and navigation. The interface with attractive color and animation would increase the motivation for learners to use CAL. The familiar icon of navigation, content with a systematic structure, and attractive menu is increasing the student's motivation using CAL. The CAL content, appearance, and navigation should be able to raise student motivation to learn; it is very important in this case because motivation is the success key of CAL to develop the self-directed learning culture.

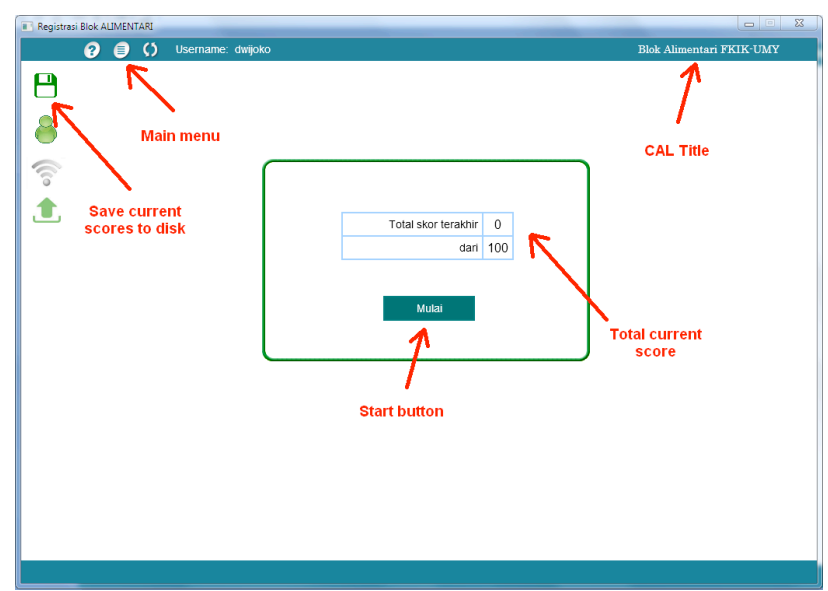

Figure 2. Starting interface

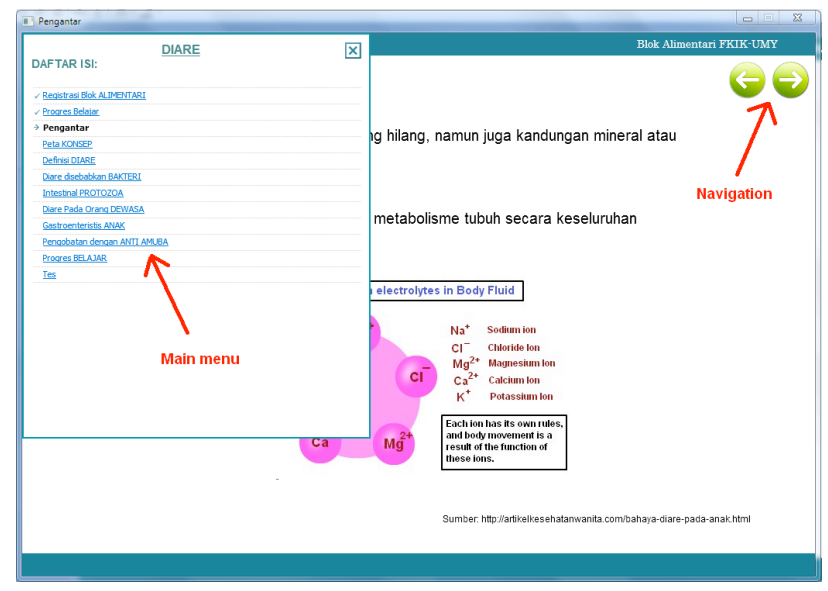

Figure 3. Learning and navigation interface

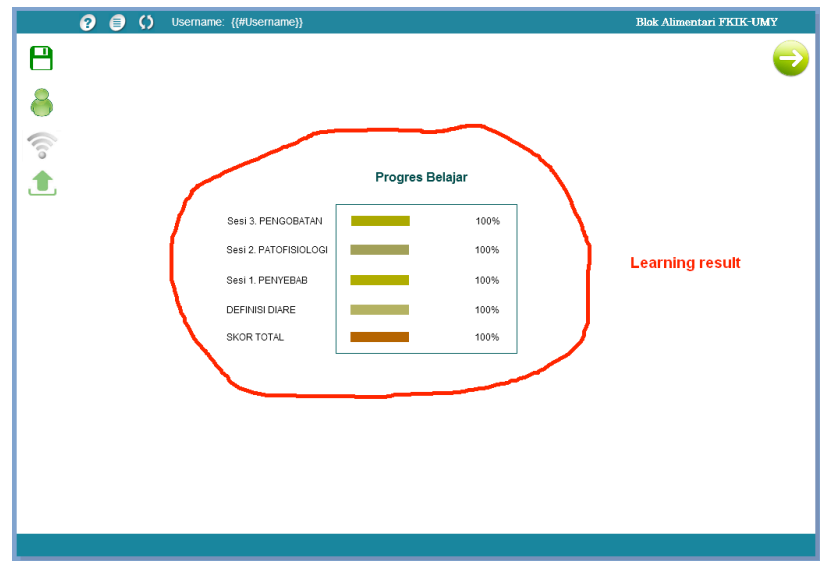

Figure 4. Learning result interface

\section{CONCLUSIONS}

The applying of Gagne's instructional design on CAL can improve the ability of learners in problem analysis, which consists of several adjusted components, these are (1) giving the clearly learning objectives, (2) explaining the objectives in the beginning, (3) giving the concept map of learning materials, (4) providing free learning sequence, (5) giving the learning content briefly, (6) describing the learning material verbally and completely, (7) presenting the learning material with animations, (8) using pictures or video. It is important to motivate 
PAPER

\section{ApPlied CAL on Problem BASEd LEARNing Using Gagne’s InStructional Design}

students by providing CAL that uses multimedia principles, convenient interface, and easy to use navigation. Beside those, for developing self-directed learning culture, it is important in each topic should provide exercises to measure the student learning progress. Finally, the alimentary CAL modules can enhance the analytical skills of students in PBL with effect size 0.89 (big effect category). It proved that the selfdirected learning culture in medical student successfully implemented. The experimental group can improve their analytical skill without any assistance or intervention by the lecturers.

\section{REFERENCES}

[1] Steinberg, E. R., Computer-assisted Instruction: a Synthesis of Theory, Practice and Technology, Lawrences Erlbaum Associates Publisher, New Jersey, 1991.

[2] Arsyad, A,. Media Pembelajaran, Penerbit PT. Raja Grafindo Persada, Jakarta, Indonesia, 2003

[3] Baddeley, A.D., Working Memory, Science Journal, 1992, pp. 556-559.

[4] Chandler, P., \& Sweller, J., The split-attention effect as a factor in the design of instruction. British Journal of Educational Psychology 62 (2), 1992, pp. 233-246. http://dx.doi.org/10.1111/j.20448279.1992.tb01017.x

[5] Sweller, J., Cognitive load theory, learning, difficulty, and instructional design. Learning and Instruction 4, 1994, pp. 295-312. http://dx.doi.org/10.1016/0959-4752(94)90003-5

[6] Paivio, A., Mental representations: a dual coding approach. Oxford University Press. Oxford, 1986.

[7] Mayer, R.E., Multimedia Learning: Are Asking the right question?. Educational Psychologist, 1997, pp. 1-19. http://dx.doi.org/10.1207/s15326985ep3201_1

[8] Paas, F, Renk1, A. \& Sweller, J., Cognitive Load Theory: Instructional Implications of the Interaction betweem Information Structures and Cognitive Architecture. Instructional Science, 32 (1-2), 2004, pp. 1-8 http://dx.doi.org/10.1023/B:TRUC.000002180 6.17516.d0

[9] Sweller, J., Implications of Cognitive Load Theory for Multimedia Learning. In R. Mayer (Ed.), Cambridge handbook of multimedia learning Cambridge University Press, New York. 2005, pp. 19-30. http://dx.doi.org/10.1017/CBO9780511816819.003

[10] Chandler, P., \& Sweller, J., The split-attention effect as a factor in the design of instruction. British Journal of Educational Psychology, 62 (2), 1992, pp. 233-246. http://dx.doi.org/10.1111/j.2044$\underline{\text { 8279.1992.tb01017.x }}$

[11] Gagne, R.M., Principles of Instructional Design. Thomson/Wordsworth, Belmonth, California, USA, 2005.

[12] Davison, R.M., Martinsons, M.G. and Kock N., Principles of Canonical Action Research, Information Systems Journal 14, 2004, pp. 65-86 http://dx.doi.org/10.1111/j.1365-2575.2004. 00162.x

[13] Mayer, R.E., Moreno, R., A., Cognitive Theory of Multimedia Learning: Implications for Design Principles, Educational Psychology, Lawrence Erlburn Association Inc., 2003.

[14] Sweller, J., Implications of Cognitive Load Theory for Multimedia Learning. In R. Mayer (Ed.), Cambridge handbook of multimedia learning Cambridge University Press, New York, 2005. pp. 19-30. http://dx.doi.org/10.1017/CBO9780511816819.003

[15] Mayer, R.E., Applying the science of learning: Evidence-based principles for the design of multimedia instruction. American Psychologist, 63(8), 2008. pp. 760-769. http://dx.doi.org/10.1037/ 0003-066X.63.8.760

\section{AUTHORS}

Sundari, S. is with the Medical Education Department, Universitas Muhammadiyah Yogyakarta, DI. Yogyakarta, 55183 Indonesia (e-mail: sundari_purbo@yahoo.mail.sg).

Harsono, was with Medical Education Department, Gadjah Mada University, DI. Yogyakarta 55281 Indonesia (e-mail: harsono_jombor@yahoo.com).

Prihatiningsih, TS. is with Medical Education Department, Gadjah Mada University, DI. Yogyakarta 55281 Indonesia (e-mail: savitri_66@yahoo.com).

Emilia, O. is with Medical Education Department, Gadjah Mada University, DI. Yogyakarta 55281 Indonesia (e-mail: ovaemilia@google.co.id).

Submited 11 August 2014. Published as resubmitted by the authors 15 October 2014. 